\title{
RETIFICADOR TRIFÁSICO ISOLADO EM ALTA FREQÜÊNCIA E COM BAIXA DISTORÇÃO DE CORRENTE NA REDE
}

\author{
Falcondes José Mendes de Seixas* e Ivo Barbi**
}

\author{
* UNESP - Universidade Estadual Paulista \\ DEE-Departamento de Engenharia Elétrica \\ 15385-000 - Cx. P. 31 - Ilha Solteira - SP \\ Telefone: (18) 3743-1150 - fax: (18) 3743-1163 \\ www.dee.feis.unesp.br - falcon@dee.feis.unesp.br
}

\author{
** UFSC - Universidade Federal de Santa Catarina \\ INEP - Instituto de Eletrônica de Potência \\ 88040-970 - Cx. P. 5119 - Florianópolis - SC \\ Telefone: (48)-331-9204 - fax: (48)-234-5422 \\ www.inep.ufsc.br - ivo@inep.ufsc.br
}

\begin{abstract}
Resumo - Um retificador trifásico de $12 \mathrm{~kW}$, com baixa distorção harmônica das correntes de linha, baseado em conexão diferencial de autotransformador de 18 pulsos e isolamento em alta freqüência, é apresentado neste trabalho. São utilizados três conversores full-bridge para permitir isolamento e equilibrar as correntes nos barramentos $\mathrm{CC}$, sem sensores ou controladores de corrente. A topologia proporciona a saída CC regulada através de uma estratégia de controle muito simples e com correção natural do fator de potência na rede trifásica de alimentação. Os resultados matemáticos, através de análise de Fourier das correntes nos enrolamentos e da análise fasorial das tensões sobre os enrolamentos são apresentados. São mostrados também resultados experimentais para validar 0 conceito apresentado.
\end{abstract}

Abstract - A 12kW three-phase rectifier with low THD in the line currents, based on an 18-pulse transformer arrangement and high-frequency isolation, is presented in this work. Three full-bridge converters are used to allow isolation and to balance the DC-link currents, without current sensing or current controller. The topology provides a regulated DC output with a very simple control strategy and natural three-phase input power factor correction. Analytical results from Fourier analysis of winding currents and the vector diagram of winding voltages are presented. Experimental results to verify the proposed concept are shown in the paper.

\section{INTRODUÇÃO}

Os mais modernos retificadores, usados principalmente em acionamentos elétricos e fontes de alimentação de telecomunicação, são projetados para drenar uma forma de onda de corrente senoidal da rede, com um fator de potência muito próximo de unitário.

Os retificadores monofásicos que satisfazem esta exigência são já bem conhecidos e muito usados. A solução padrão utiliza um pré-regulador de fator de potência baseado no conversor boost PWM alimentado por um retificador de onda completa a diodos. Porém, em aplicações de média potência (6kW ou mais), a solução monofásica não é conveniente, ficando então o retificador trifásico como melhor opção.
Da mesma forma que um grande número de trabalhos foi desenvolvido para correção de fator de potência em sistemas monofásicos, as técnicas trifásicas estão em constante crescimento [1]. Este crescimento também aplica-se a conversores com um ou mais interruptores associados, ou usando transformadores especialmente conectados ou sistemas mistos com transformadores e conversores estáticos.

A solução mais simples usa um retificador trifásico a diodos, associado a filtros passivos para minimizar os componentes harmônicos das correntes de linha. O Isolamento pode ser obtido usando um transformador convencional de baixa freqüência $\Delta / Y$, resultando em um equipamento volumoso, pesado e caro. De forma contrária, encontra-se o retificador PWM trifásico clássico, que requer um circuito de controle bem mais complexo, modulação e técnicas de comutação mais sofisticadas.

Se o isolamento não é importante para o projeto, algumas soluções que utilizam arranjos de transformador [2-5] ou transformadores de inter-fase de linha (LIT- line interphase transformer) $[6,7]$ são muito importantes para melhorar a qualidade das correntes da rede. Estes transformadores apresentam uma reduzida potência aparente (kVA). O conversor de 18-pulsos que utiliza um autotransformador diferencial conectado em $\mathrm{Y}$ ou $\Delta$, é muito interessante porque permite a correção natural do fator de potência (os menores componentes harmônicos são o $17^{\circ}$ e o $19^{\circ}$ ). O autotransformador é projetado para alimentar três retificadores de seis pulsos, defasados de $20^{\circ}$ e processando aproximadamente $20 \%$ dos kVA necessários. Normalmente, para realizar a conexão paralela das tensões retificadas, dois transformadores de interfase (IPT), conectados nos lados CC dos três retificadores em ponte, são necessários para absorver as diferenças instantâneas de tensão entre as pontes.

Sempre que o isolamento e a regulação da tensão de saída são requeridos, como em sistemas de telecomunicação, o desafio é encontrar um conversor trifásico robusto com alto rendimento, alta densidade de potência e baixo custo.

Este trabalho apresenta um retificador de 18 pulsos isolado e com saída CC regulada de 60V/200A [8-10]. A técnica usa o mesmo conceito do autotransformador polifásico para obter a correção natural do fator de potência. Além disso, inclui o isolamento em alta freqüência e permite a regulação da tensão de saída com baixa ondulação, sem utilizar malhas de controle de corrente. A técnica de comutação ZVS-PWM para os interruptores ativos é aplicada a esta topologia. A 
conexão proposta para os transformadores de alta freqüência elimina os transformadores de interfase. Assim, o tamanho final do conversor e a complexidade do circuito de comando ficam reduzidos.

\section{TOPOLOGIA DO CONVERSOR}

O conceito fundamental da correção natural do fator de potência através de arranjos de transformadores ou de autotransformadores é assegurado pelo autotransformador de 18 pulsos conectado em Y, seguido de três retificadores de seis pulsos a diodos.

A topologia do conversor proposto é mostrada na Fig. 1. Esta solução usa três conversores full-bridge conectados nos lados CC de cada retificador trifásico. Um filtro de alta freqüência e pequeno volume $\left(\mathrm{L}_{\mathrm{f}}, \mathrm{C}_{\mathrm{f}}\right)$ é conectado em cada saída CC (entre os conversores full-bridge e os retificadores trifásicos).

Além dos transformadores de alta freqüência, que permitem isolamento entre os lados primários e secundários, os enrolamentos secundários estão conectados em série para equilibrar as correntes dos lados $\mathrm{CC}$ dos retificadores. Esta estratégia simples e robusta elimina todos os sensores e os controladores de corrente, que geralmente são necessários para equilibrar as correntes nas saídas dos retificadores. Porém, os conversores full-bridge têm que ser sincronizados. Com o objetivo de reduzir as perdas de comutação, sem utilizar interruptores auxiliares, a técnica PWM com deslocamento de fase (phase-shift) é aplicada. Os componentes ressonantes, snubbers e circuitos grampeadores de tensão não são mostrados na Fig. 1 .

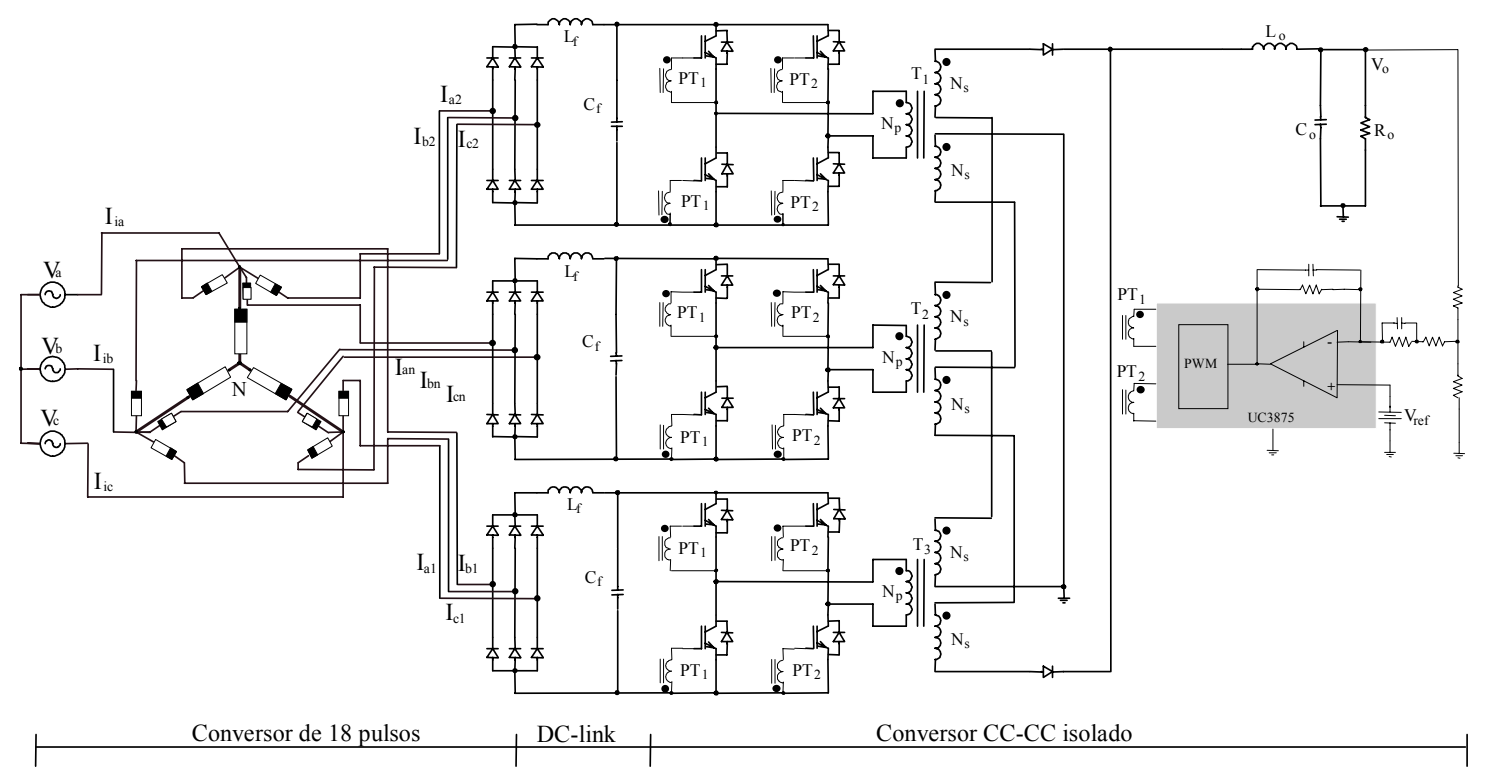

Fig. 1. Retificador trifásico proposto com isolamento em alta freqüência.

A tensão de saída regulada é obtida facilmente por um controlador de tensão convencional. Apenas um circuito integrado (PS-PWM) [11], associado a alguns componentes passivos e dois transformadores de pulso (PT1 e PT2), são usados para regulação e comando dos interruptores. Através dos transformadores de pulso, a sincronização entre os conversores full-bridge é facilmente conseguida.

\section{A. Análise do Autotransformador}

Os enrolamentos primários do autotransformador são formados por $\mathrm{N}_{\mathrm{a}}, \mathrm{N}_{\mathrm{b}}$ e $\mathrm{N}_{\mathrm{c}}$ que estão conectados em $\mathrm{Y}$, submetidos às tensões de linha $\mathrm{V}_{\mathrm{a}}, \mathrm{V}_{\mathrm{b}} \mathrm{e} \mathrm{V}_{\mathrm{c}}$. Nesta conexão, um neutro virtual $\mathrm{N}$ é gerado.

Os enrolamentos secundários são projetados, de tal modo que, o número de espiras e a conexão entre eles e o enrolamentos primário geram três sistemas trifásicos diferentes, defasados de $20^{\circ}$ um do outro. Estes três sistemas de tensão é que alimentam os três retificadores.

Todos os enrolamentos $\mathrm{N}_{\mathrm{a}}, \mathrm{N}_{\mathrm{a} 1}, \mathrm{~N}_{\mathrm{a} 2}$ e $\mathrm{N}_{\mathrm{an}}$ são acoplados na mesma perna do núcleo, as tensões resultantes $\mathrm{V}_{\mathrm{a}}, \mathrm{V}_{\mathrm{a} 1}, \mathrm{~V}_{\mathrm{a} 2} \mathrm{e}$ $\mathrm{V}_{\mathrm{an}}$, estão em fase. $\mathrm{O}$ mesmo se aplica às fases " $\mathrm{b}$ " $\mathrm{e}$ "c".
Uma representação esquemática dos enrolamentos primário e secundários, das conexões elétricas e do núcleo trifásico é mostrada na Fig. 2.

1) Tensões sobre os enrolamentos - O autotransformador é alimentado por um sistema trifásico de tensões balanceadas. Três retificadores a diodos são alimentados pelas tensões secundárias, compostas de três sistemas de tensão trifásicos, também equilibrados. Um destes sistemas é colocado em fase como a tensão da rede e os outros são defasados de $+20^{\circ}$ e de $-20^{\circ}$, com relação a este sistema. O diagrama fasorial e o triângulo auxiliar, usado para obter os três sistemas de tensão, são mostrados na Fig. 3. 


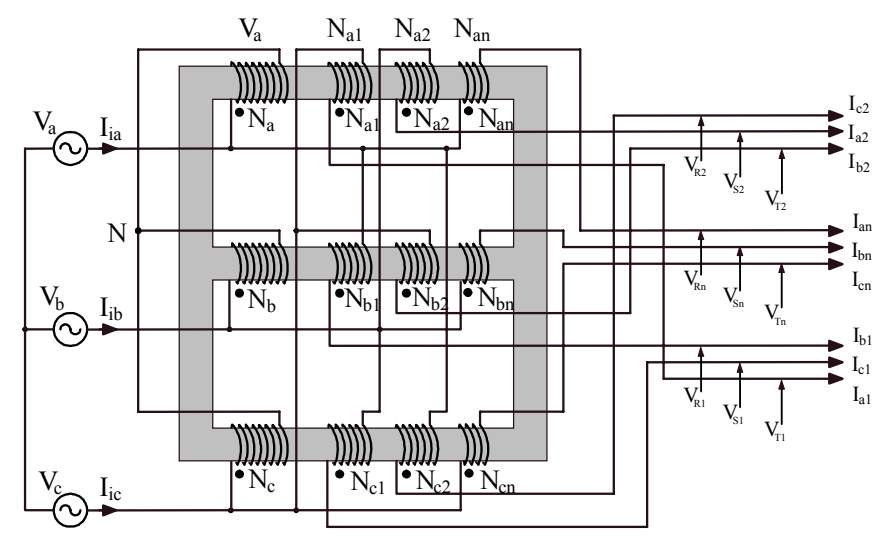

Fig. 2. Esquema dos enrolamentos do autotransformador.

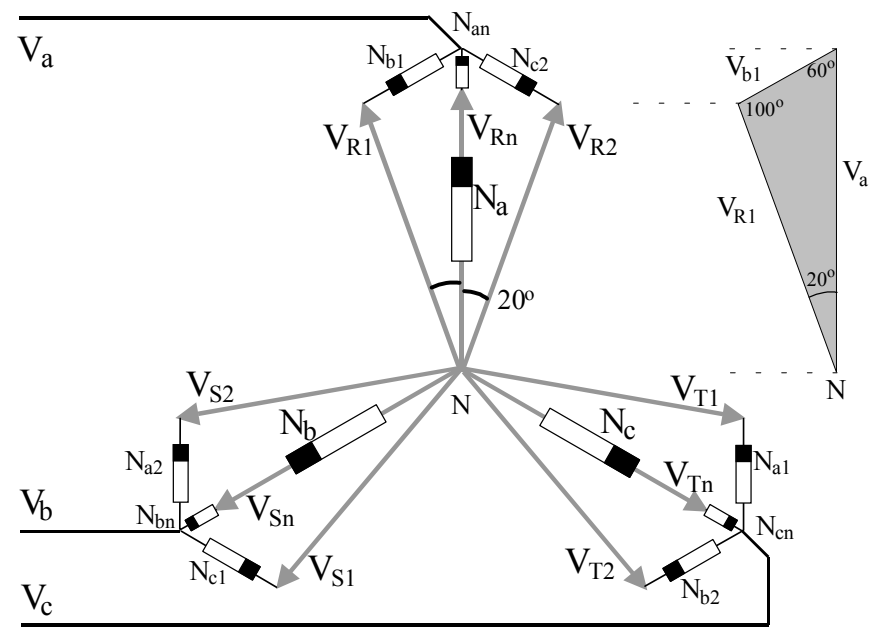

Fig. 3. Diagrama fasorial e triângulo auxiliar.

As amplitudes das tensões através dos enrolamentos secundários $\mathrm{V}_{\mathrm{a} 1}, \mathrm{~V}_{\mathrm{a} 2}, \mathrm{~V}_{\mathrm{b} 1}, \mathrm{~V}_{\mathrm{b} 2}, \mathrm{~V}_{\mathrm{c} 1}, \mathrm{e} \mathrm{V}_{\mathrm{c} 2}$ são obtidas por (1).

$$
V_{b 1}=V_{a} \cdot \frac{\operatorname{sen}\left(20^{\circ}\right)}{\operatorname{sen}\left(100^{\circ}\right)}=0,35 \cdot V_{a}
$$

A relação de espiras $\left(\mathrm{K}_{1}\right)$ que assegura um deslocamento de fase de $20^{\circ}$ é determinada por (2).

$$
K_{1}=\frac{V_{a}}{V_{b 1}}=2,88
$$

Este resultado mostra que o número de espiras destes secundários são 2,88 vezes menores que o número de espiras do primário.

As amplitudes das tensões entre cada par de terminais dos secundários, $\left(\mathrm{V}_{\mathrm{R} 1}, \mathrm{~V}_{\mathrm{S} 1}, \mathrm{~V}_{\mathrm{T} 1}\right)$ e $\left(\mathrm{V}_{\mathrm{R} 2}, \mathrm{~V}_{\mathrm{S} 2}, \mathrm{~V}_{\mathrm{T} 2}\right)$, com respeito ao neutro virtual, são obtidas pela expressão (3).

$$
V_{R I}=V_{a} \cdot \frac{\operatorname{sen}\left(60^{\circ}\right)}{\operatorname{sen}\left(100^{\circ}\right)}=0,88 \cdot V a
$$

$\mathrm{O}$ terceiro sistema trifásico de tensão dos secundários $\left(\mathrm{V}_{\mathrm{Rn}}\right.$, $\mathrm{V}_{\mathrm{Sn}}, \mathrm{V}_{\mathrm{Tn}}$ ) está em fase com o primário. Estas tensões, porém, tem que ter as mesmas amplitudes dos outros secundários. Assim, a equação (4) deve ser cumprida.

$$
V_{R n}=V_{a}-0,88 \cdot V_{a}=0,12 \cdot V_{a}
$$

A relação de espiras que assegura $88 \%$ da tensão primária $\left(\mathrm{K}_{2}\right)$, sem deslocamento de fase, é determinada por (5).

$$
K_{2}=\frac{V_{a}}{V_{R n}}=8,29
$$

Este resultado mostra que o número de espiras dos enrolamentos secundários é 8,29 vezes menor que do primário.

Pode-se observar que as amplitudes de tensão dos três sistemas trifásicos são aproximadamente $88 \%$ dos valores das tensões de fase da rede.

2) Correntes nos enrolamentos - A técnica para eliminar componentes harmônicos de corrente em conversores de múltiplos pulsos requer operação da carga no modo de fonte de corrente. O conversor de 18 pulsos é obtido quando cada um dos sistemas de tensão de saída é conectado a um retificador a diodo de seis pulsos. É como se três cargas idênticas (I/3), com características de fonte de corrente, fossem utilizadas.

A forma de onda de corrente, através de um enrolamento secundário $\left(\mathrm{N}_{\mathrm{an}}\right)$, em fase com a tensão da rede $\mathrm{V}_{\mathrm{a}}$, é mostrada na Fig. 4. Esta forma de onda é adotado como uma referência angular para representar as correntes dos outros enrolamentos.

A forma de onda de $\mathrm{I}_{\mathrm{an}}$ pode ser decomposta em uma série de Fourier através da maneira convencional. No entanto, quando se trata de uma função descontínua, os termos da série podem ser obtidos por inspeção. Pode-se observar que esta forma de onda apresenta simetria alternada, o semi-ciclo negativo é uma reprodução invertida do semi-ciclo positivo. Assim, os componentes harmônicos pares são nulos e não há nenhum termo em cosseno. $\mathrm{O}$ valor médio também é zero.

Note que o enrolamento $\mathrm{N}_{\mathrm{an}}$ conduz a corrente $\mathrm{I} / 3$ durante $120^{\circ}(2 \pi / 3)$, a partir de $30^{\circ}(\pi / 6)$. Assim, a expressão de corrente resulta em (6).

$$
I_{a n}(t)=\frac{4}{\pi} \cdot \frac{I}{3} \cdot \sum_{k} \frac{1}{k} \cdot \cos \left(k \cdot \frac{1}{6}\right) \cdot \operatorname{sen}(k \cdot \omega t)
$$

Onde, $\mathrm{k}=1,3,5, \ldots$

As formas de onda de corrente que circulam pelos outros enrolamentos secundários deste sistema trifásico $\left(\mathrm{I}_{\mathrm{bn}}\right.$ e $\left.\mathrm{I}_{\mathrm{cn}}\right)$ são representadas pela mesma equação de $\mathrm{I}_{\mathrm{an}}$. No entanto, as fases são deslocadas de $-120^{\circ} \mathrm{e}+120^{\circ}$.

No outro sistema secundário trifásico, (defasado de $+20^{\circ}$ ), a corrente $\mathrm{I}_{\mathrm{b} 1}$ é expressa por (7).

$$
I_{b 1}(t)=\frac{4}{\pi} \cdot \frac{I}{3} \cdot \sum_{k} \frac{1}{k} \cdot \cos \left(k \cdot \frac{1}{6}\right) \cdot \operatorname{sen}\left[k \cdot\left(\omega t+20^{\circ}\right)\right]
$$

As outras correntes deste sistema trifásico $\left(\mathrm{I}_{\mathrm{a} 1} \quad \mathrm{e} \mathrm{I}_{\mathrm{c} 1}\right)$ são representadas pela mesma equação de $\mathrm{I}_{\mathrm{b} 1}$, porém, deslocadas de $-120^{\circ}$ e $+120^{\circ}$.

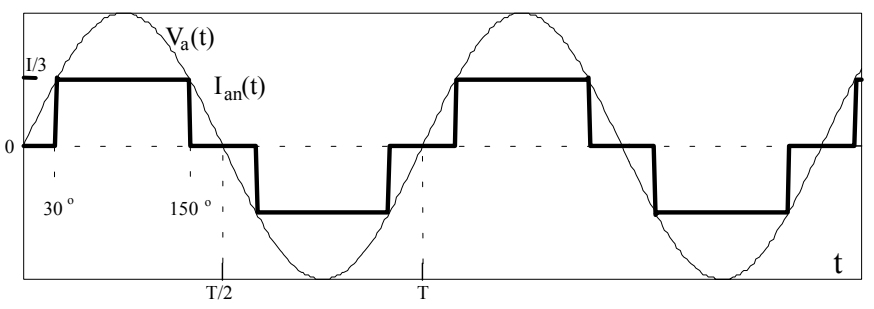

Fig. 4. Tensão primária e corrente secundária para a fase "a".

Para o último sistema de tensão (deslocado de $-20^{\circ}$ ), a 
corrente $\mathrm{I}_{\mathrm{c} 2}$ é expressa por (8).

$$
I_{c 2}(t)=\frac{4}{\pi} \cdot \frac{I}{3} \cdot \sum_{k} \frac{1}{k} \cdot \cos \left(k \cdot \frac{1}{6}\right) \cdot \operatorname{sen}\left[k \cdot\left(\omega t-20^{\circ}\right)\right]
$$

As outras correntes deste sistema trifásico $\left(\mathrm{I}_{\mathrm{a} 2}\right.$ e $\left.\mathrm{I}_{\mathrm{b} 2}\right)$ são representadas pela mesma equação de $\mathrm{I}_{\mathrm{c} 2}$, porém, deslocadas de $-120^{\circ}$ e $+120^{\circ}$.

As correntes dos enrolamentos primários $\left(\mathrm{I}_{\mathrm{a}}, \mathrm{I}_{\mathrm{b}}\right.$ e $\left.\mathrm{I}_{\mathrm{c}}\right)$ podem ser obtidas pela expressão (9), considerando as correntes dos três enrolamentos secundários que estão montados sobre a mesma perna do núcleo e com relações de espira $\mathrm{K}_{1}$ e $\mathrm{K}_{2}$. Como mencionado, os enrolamentos com os mesmos índices ( $\mathrm{a}, \mathrm{b}$ ou c) são montados sobre uma mesma perna do núcleo. A Fig. 5 mostra a forma de onda da corrente $\left(\mathrm{I}_{\mathrm{a}}\right)$ do primário.

$$
I_{a}(t)=\left(\frac{I_{a 1}(t)+I_{a 2}(t)}{K_{1}}-\frac{I_{a n}(t)}{K_{2}}\right)
$$

3) correntes na rede - As correntes na rede $\mathrm{I}_{\mathrm{ia}}, \mathrm{I}_{\mathrm{ib}}$ e $\mathrm{I}_{\mathrm{ic}}$ são obtidas somando todas as correntes dos enrolamentos que chegam num mesmo nó. Então, a equação para $\mathrm{I}_{\text {ia }}$ pode ser representada por (10).

$$
I_{i a}(t)=I_{a}(t)+I_{a n}(t)+I_{b 1}(t)+I_{c 2}(t)
$$

A Fig. 6 mostra as correntes de linha $\left(\mathrm{I}_{\mathrm{ia}}, \mathrm{I}_{\mathrm{ib}}\right.$ e $\left.\mathrm{I}_{\mathrm{ic}}\right)$ e a Fig. 7 mostra o espectro harmônico da corrente $\mathrm{I}_{\mathrm{ia}}$ em valores (\%) da amplitude do componente fundamental. Pode-se observar que o conversor de 18 pulsos apresenta apenas os componentes harmônicos de ordens $\mathrm{k} .18 \pm 1$, para $\mathrm{k}=1,2$, $3, \ldots$ e as amplitudes são menores que $6 \%$ do componente fundamental $\left(\mathrm{I}_{\mathrm{ia}}\right)$.

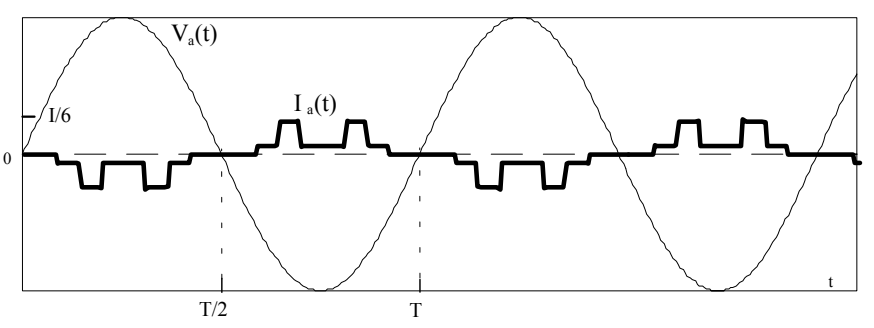

Fig. 5. Corrente primária para a fase "a".

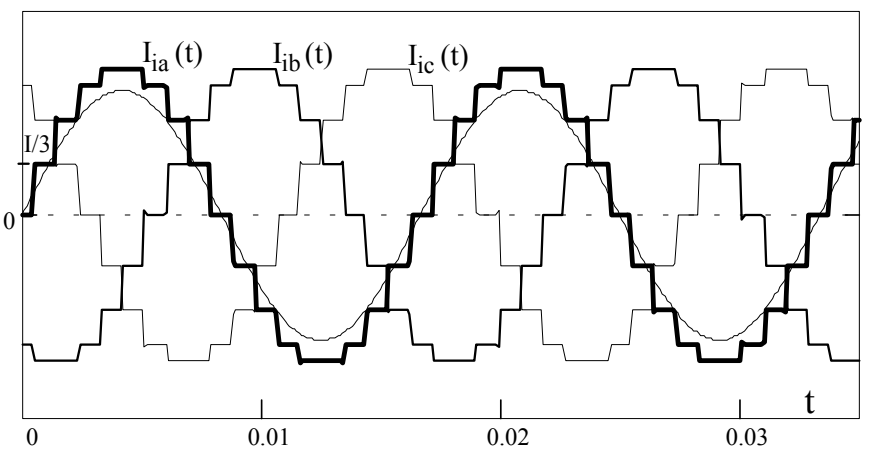

Fig. 6. Correntes de linha e tensão da fase "a".

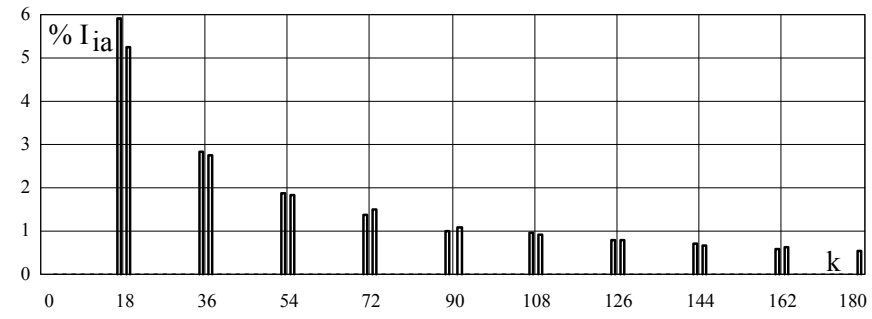

Fig. 7. Harmônicos da corrente $\mathrm{I}_{\mathrm{ia}}$.

\section{B. Conversor CC-CC isolado}

A topologia do conversor CC-CC isolado a ser escolhida, deve ser de um conversor alimentado em corrente de baixa ondulação, conectado nas saídas dos retificadores, ou seja, três conversores CC-CC que devem absorver as correntes dos barramentos $\mathrm{CC}$ de forma equilibrada e com baixa ondulação. Assim, a classe dos conversores isolados alimentados em corrente (boost) como o push-pull e o fullbridge são os mais atraentes.

$\mathrm{O}$ equilíbrio das correntes pode ser alcançado através do controle de corrente, monitorando-se as correntes dos três barramentos CC por sensores de corrente. Assim, um regulador de tensão que gera apenas uma referência de corrente para os três reguladores pode controlar a tensão de saída [4].

Neste trabalho, a estratégia para equilibrar todas as correntes dos barramentos $\mathrm{CC}$ não utiliza qualquer sensor ou controlador de corrente. A própria topologia do circuito de potência garante o equilíbrio das correntes, como descrito a seguir:

1) Topologia do conversor - A topologia escolhida para o estágio isolado foi de um conversor full-bridge alimentado em tensão com um filtro LC de entrada. Esta topologia alimentada em tensão permite empregar a técnica de modulação por largura de pulso e deslocamento de fase (PWM-PS). Então, não há sobre-tensão nos interruptores e a comutação com tensão zero (ZVS) fica garantida para uma larga faixa de operação [11]. Os componentes ressonantes LC incorporam as capacitâncias de saída dos interruptores e as indutâncias de dispersão dos enrolamentos primários.

Um pequeno filtro LC (que opera em duas vezes a freqüência de chaveamento) é instalado na entrada de cada conversor $\mathrm{CC}-\mathrm{CC}$ para filtrar os componentes de alta freqüência das correntes nos três barramentos CC-CC.

2) Equilibro das correntes - Os três conversores CC-CC apresentam as seguintes características:

- Processam a mesma potência ( $1 / 3$ da potência total);

- Os sistemas de tensões retificadas (6 pulsos) têm mesmas amplitudes, embora deslocados de $20^{\circ}$;

- As correntes médias nos três barramentos CC são iguais.

O balanço de corrente pode ser alcançado pela conexão série dos enrolamentos secundários dos três transformadores de alta freqüência e pelo sincronismo do comando dos conversores. Assim, as formas de onda de corrente dos enrolamentos secundários são iguais e, devido à relação de espiras dos transformadores, todas as correntes pelos enrolamentos primários são idênticas $\left(\mathrm{I}_{\mathrm{p} 1}=\mathrm{I}_{\mathrm{p} 2}=\mathrm{I}_{\mathrm{p} 3}\right)$, como mostrado na Fig. 8. 


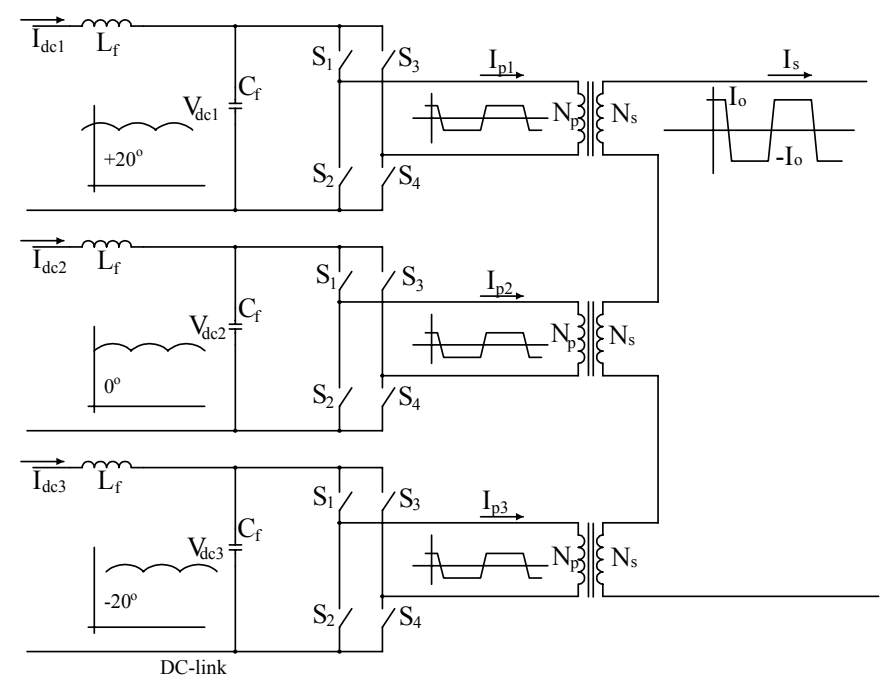

Fig. 8. Conexão para equilíbrio das correntes nos barramentos CC.

Como conseqüência, as correntes instantâneas pelos três conversores são iguais. Devido às diferenças instantâneas entre as tensões retificadas, as potências processadas pelos três conversores CC-CC, durante um período de chaveamento, também são diferentes. Assim, a freqüência da ondulação das correntes nos barramentos CC é de três vezes a freqüência da ondulação de cada tensão retificada. Este efeito é resultado da composição das três tensões retificadas (6 pulsos) com um deslocamento de $20^{\circ}$. A Fig. 8 mostra a estratégia para alcançar o equilíbrio das correntes que circulam através dos barramentos $\mathrm{CC}$.

3) Retificador de saída - Para reduzir as perdas de condução dos diodos, a conexão de ponto médio é escolhida para o retificador de saída. Assim, cada transformador passa a ter dois enrolamentos secundários que são conectados como mostrado na Fig. 1. A tensão a ser retificada resulta da soma das tensões secundárias.

Cada tensão secundária, cuja fase corresponde à fase da tensão do respectivo barramento $\mathrm{CC}$, apresenta uma ondulação de seis pulsos. Então, como resultado, a tensão de saída apresenta uma ondulação de 18-pulsos, composta pelas três tensões secundárias defasadas de $20^{\circ}$.

4) Estratégia de comando - Na Fig. 1 está mostrado o circuito de comando usado para conseguir obter a saída CC regulada e a sincronização dos três conversor full-bridge. Apenas um circuito integrado é necessário para executar o comando dos interruptores (drive) e a regulação de tensão.

\section{RESULTADOS EXPERIMENTAIS}

\section{A. Especificações e principais componentes}

A Fig. 9 mostra uma foto do protótipo completo do conversor de CA-CC trifásico implementado.

- Tensões da rede trifásica: $220 / 380 \mathrm{~V}$.

- Saída CC: 60V / 200A.

- Freqüência de chaveamento: $\mathrm{f}_{\mathrm{s}}=30 \mathrm{kHz}$.

- $\mathrm{N}_{\mathrm{a}}, \mathrm{N}_{\mathrm{b}}, \mathrm{N}_{\mathrm{c}}=330$ voltas com um fio 20AWG.

- $\mathrm{N}_{\mathrm{an}}, \mathrm{N}_{\mathrm{bn}}, \mathrm{N}_{\mathrm{cn}}=40$ voltas com um fio 15AWG.

- $\mathrm{N}_{\mathrm{a} 1}, \mathrm{~N}_{\mathrm{b} 1}, \mathrm{~N}_{\mathrm{c} 1}=114$ voltas com um fio 15AWG.

- $\quad \mathrm{N}_{\mathrm{a} 2}, \mathrm{~N}_{\mathrm{b} 2}, \mathrm{~N}_{\mathrm{c} 2}=114$ voltas com um fio 15AWG.
- Autotransformador: área do núcleo EI $=27 \mathrm{~cm}^{2}$.

- Pontes trifásicas: SKD 30/08 A1 (Semikron).

- $\mathrm{L}_{\mathrm{F}}$ e $\mathrm{C}_{\mathrm{F}}=4 \mathrm{mH}$ e $1,3 \mathrm{~F} \mu \mathrm{F}$

- Módulos de IGBT: SK 25 GH 063 (Semikron).

- Diodos retificadores: HFA50PA60C (IR).

- $\mathrm{N}_{\mathrm{p}}=13$ voltas com 16 fios 23AWG.

- $\mathrm{N}_{\mathrm{s}}=1$ volta com 150 fios $23 \mathrm{AWG}$.

- Transformador de alta freqüência: EE-65/65 - ferrite.

- $\quad$ PWM-PS = UC3875 (Texas Instruments).

- $\mathrm{L}_{\mathrm{o}}=2 \times 7,5 \mu \mathrm{H}$ : dois indutores com núcleos EE-65/39 - ferrite - 4 voltas com 100 fios 20AWG.

- $\mathrm{C}_{\mathrm{o}}=6 \times 680 \mu \mathrm{F} / 100 \mathrm{~V}$ - capacitor eletrolítico.

- Peso total $=26 \mathrm{~kg}$.

- Peso do autotransformador $=15 \mathrm{~kg}$.

- Dimensões do conversor CC-CC $=80 \mathrm{~cm}$ x $40 \mathrm{~cm}$

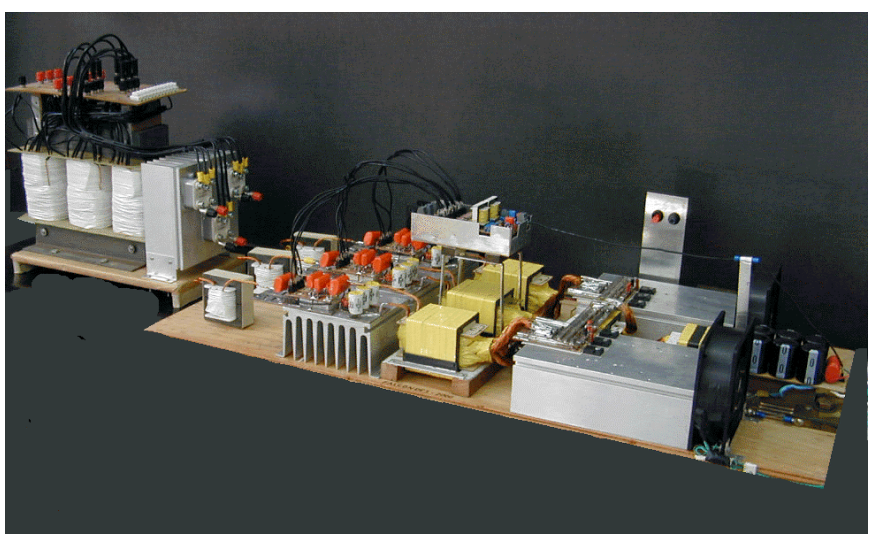

Fig. 9. Protótipo do conversor CA-CC de 12kW implementado.

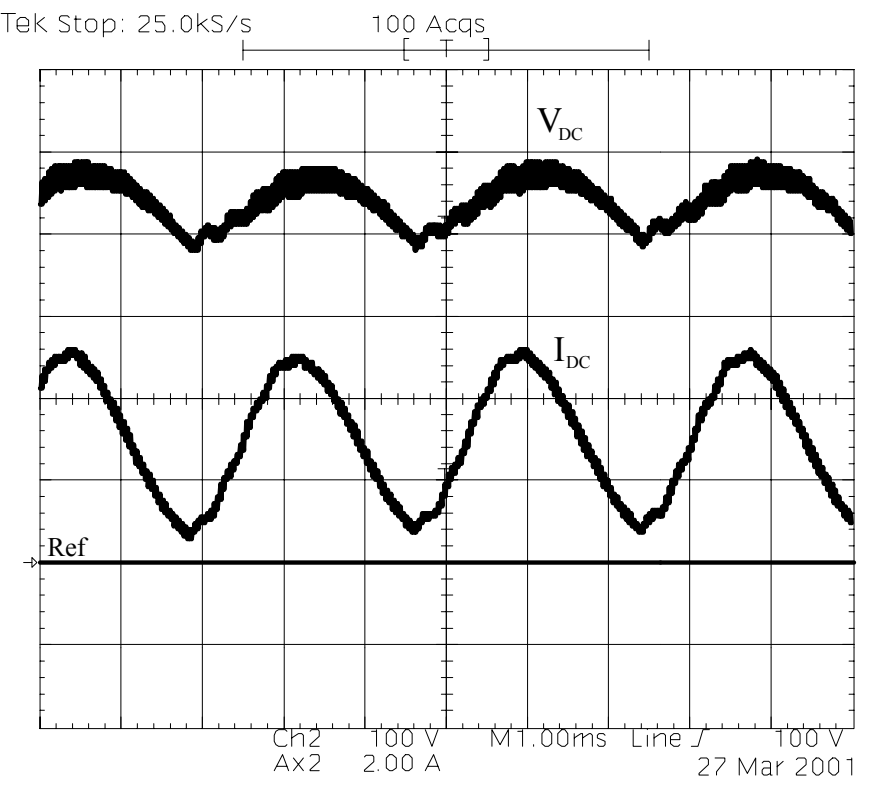

Fig. 10. Tensão e corrente no barramento CC sem conexão série dos enrolamentos secundários.

Escalas: 1ms/div, 100V/div, 2A/div.

B. Resultados experimentais

A Fig. 10 mostra as formas de onda da tensão e da corrente 
num dos barramentos CC com baixa carga para operação sem conexão série dos enrolamentos secundários. Observase a elevada amplitude da ondulação de seis pulsos da corrente. Neste modo de operação não é possível reduzir todos os componentes harmônicos de baixa ordem da linha.

As três correntes equilibradas dos barramentos CC, com os enrolamentos secundários em série, são mostradas na Fig. 11. Neste caso, são minimizadas as ondulações de baixa freqüência e as correntes médias dos barramentos $\mathrm{CC}$ são iguais.

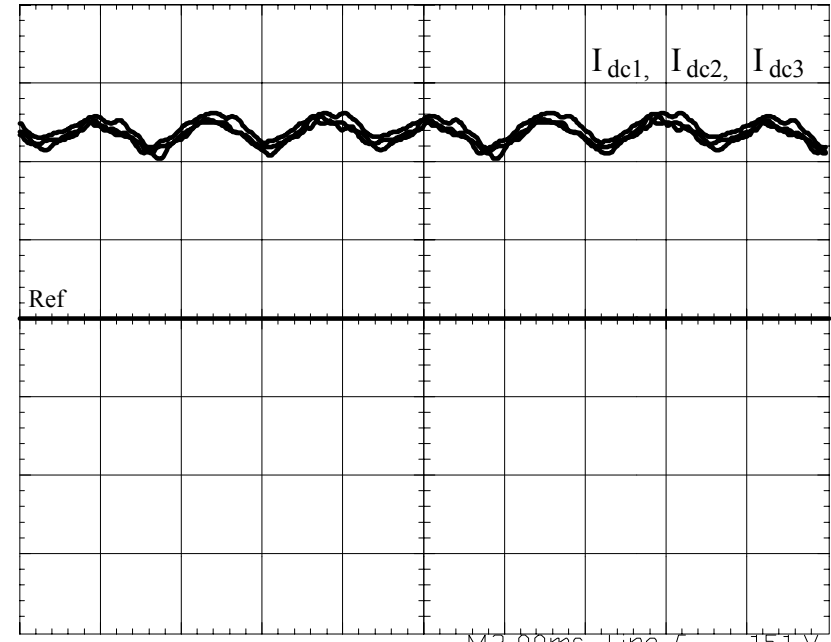

Fig. 11. Correntes equilibradas nos barramento CC. Escalas: $2 \mathrm{~ms} / \mathrm{div}, 5 \mathrm{~A} / \mathrm{div}$.

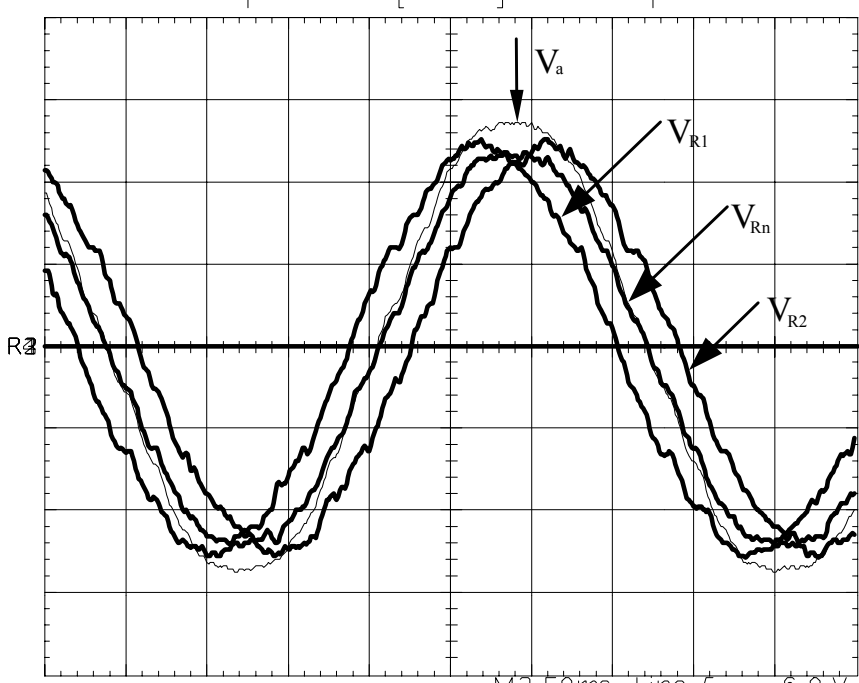

Fig. 12. Tensões nas entradas dos retificadores e na rede. Escalas: $2.5 \mathrm{~ms} / \mathrm{div}, 100 \mathrm{~V} / \mathrm{div}$.

As formas de onda das tensões de entrada de todos os retificadores e a tensão de linha, para uma fase, são mostradas na Fig. 12 e as formas de onda das tensões retificadas que alimentam os conversores full-bridge são mostradas na Fig. 13. Em ambas podem-se observar o deslocamento de fase de $20^{\circ}$ e o equilíbrio de amplitude entre elas.

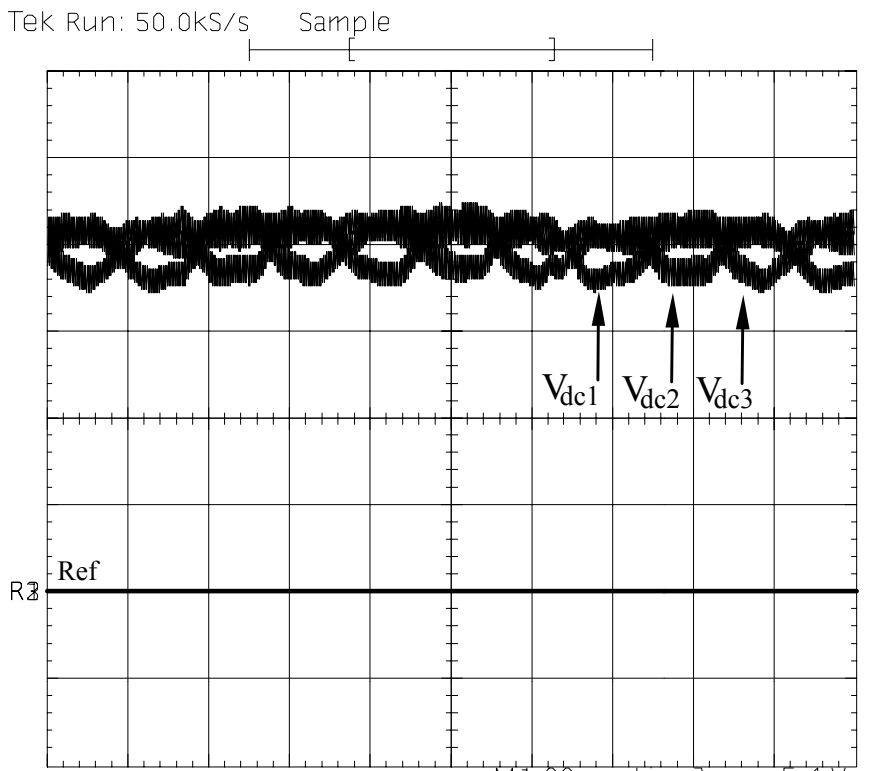

Fig. 13. Tensões retificadas nos barramentos CC. Escalas: $1 \mathrm{~ms} / \mathrm{div}, 100 \mathrm{~V} / \mathrm{div}$.
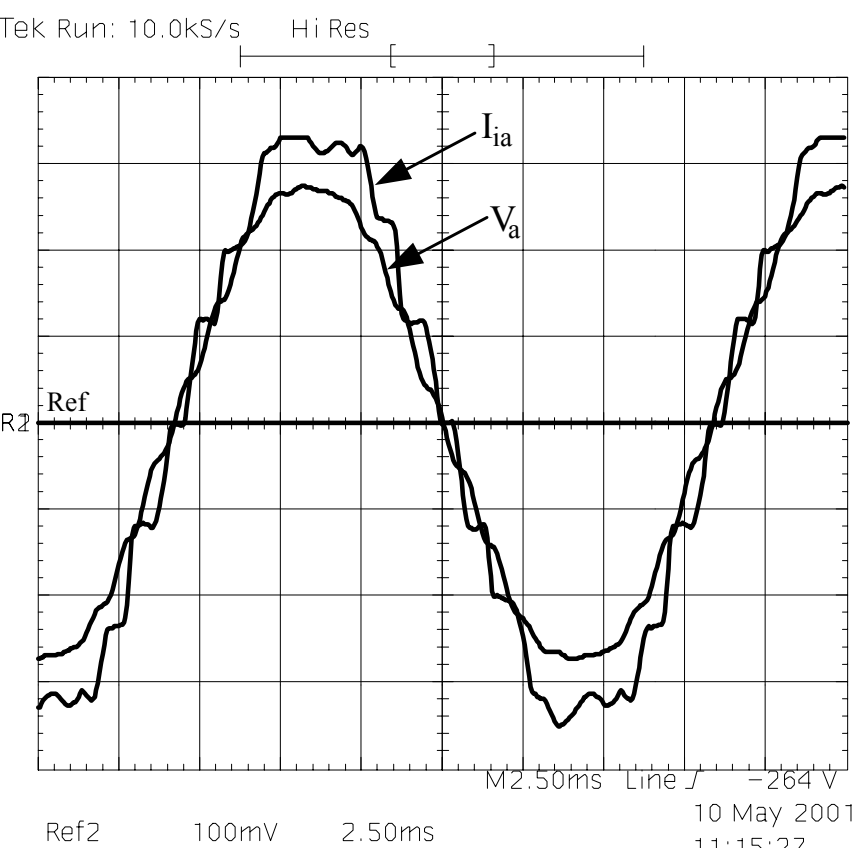

Fig. 14. Corrente e tensão da rede na fase "a". Escalas: $2 \mathrm{~ms} / \mathrm{div}, 10 \mathrm{~A} / \mathrm{div}, 100 \mathrm{~V} / \mathrm{div}$.

A Fig. 14 mostra as formas de onda de tensão e de corrente de entrada para uma das fases e a Fig. 15 mostra as três correntes de linha $\left(\mathrm{I}_{\mathrm{ia}}, \mathrm{I}_{\mathrm{ib}}\right.$ e $\left.\mathrm{I}_{\mathrm{ic}}\right)$. Pode-se observar que as 
formas das correntes de entrada para o resultado experimental (Fig. 15) e para o resultado matemático (Fig. 6) são aproximadamente iguais. Os valores medidos para o fator de potência e a THD da corrente de entrada são iguais a 0.99 e $8,6 \%$, respectivamente. A Fig. 16 mostra o espectro harmônico da corrente em uma das fases e o espectro harmônico da tensão da respectiva fase.

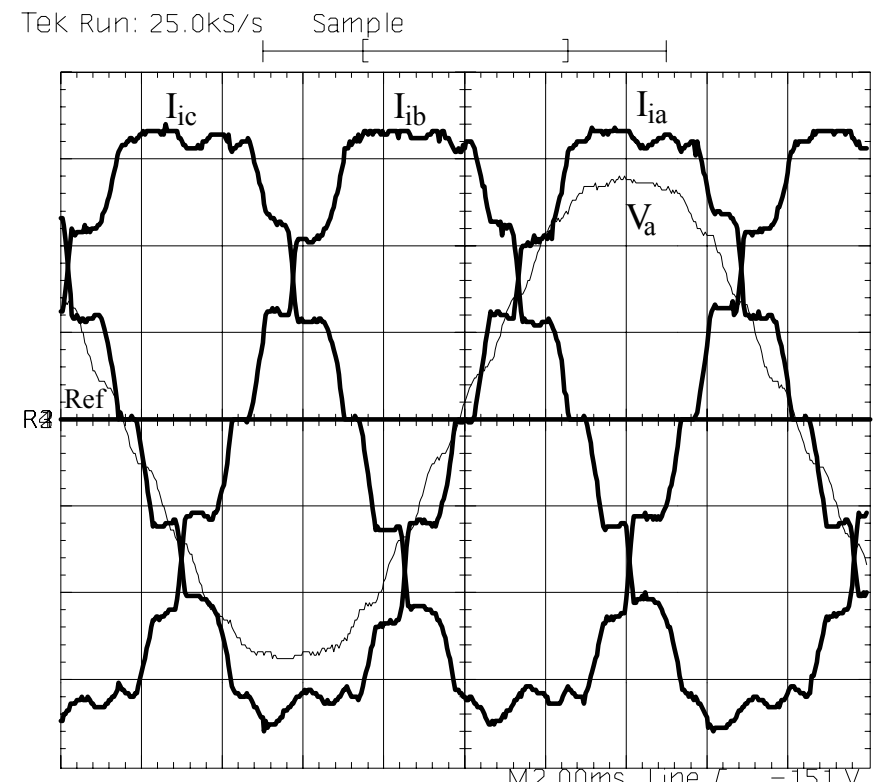

Fig. 15. Correntes na rede.

Escalas: 2ms/div, 10A/div, 100V/div.
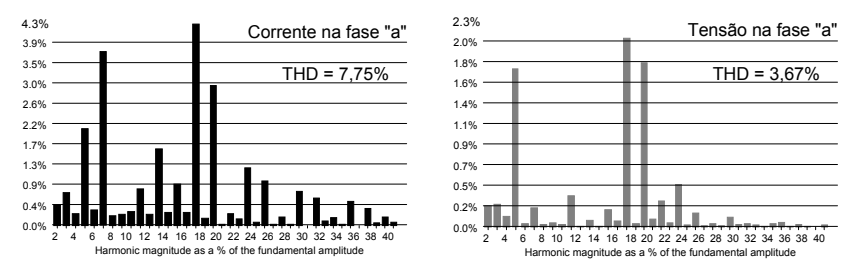

Fig. 16. Harmônicos da corrente e da tensão de uma das fases.

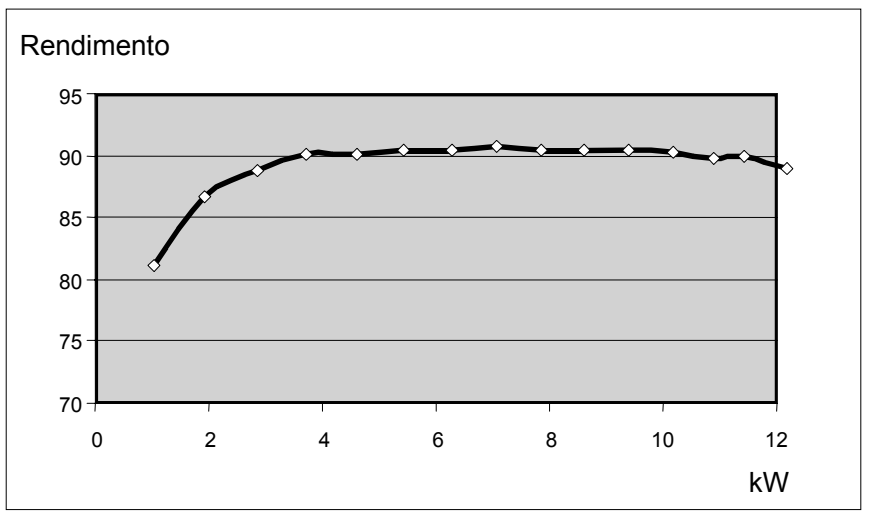

Fig. 17. Rendimento.

A Fig. 17 mostra a curva do rendimento para operação desde baixa carga até plena carga. Pode-se observar que o rendimento é superior a $90 \%$, a partir de $1 / 3$ da carga nominal.

\section{CONCLUSÕES}

Neste trabalho é apresentado um retificador trifásico isolado e com baixa distorção de corrente na rede. $O$ conversor de 18 pulsos é baseado em um autotransformador diferencial, conectado em Y, seguido de três retificadores a diodos e três conversores full-bridge (ZVS-PWM-PS). Os enrolamentos secundários dos transformadores de alta freqüência são conectados em série e o comando dos três conversores full-bridge são sincronizados para que as correntes nos barramentos $\mathrm{CC}$ fiquem equilibradas. $\mathrm{O}$ equilíbrio e a baixa ondulação na amplitude das correntes dos barramentos são a exigência fundamental para haver redução no conteúdo harmônico de corrente na rede. Um protótipo de laboratório de $12 \mathrm{~kW}$ foi implementado e os resultados experimentais completos estão apresentados. Tratando-se de uma topologia isolada, o rendimento obtido foi excelente, podendo ainda ser melhorado através da redução de perdas de condução, tanto nos semicondutores como no cobre dos diversos enrolamentos. A simplicidade, robustez e alta densidade de potência do conversor proposto coloca-o como forte candidato para soluções modernas para sistemas retificadores trifásicos utilizados em sistemas de alimentação para telecomunicações e também para acionamentos.

\section{REFERÊNCIAS BIBLIOGRÁFICAS}

[1] J. W. Kolar, "Status of the Techniques of Three-Phase Rectifier Systems with Low Effects on the Mains", in INTELEC Conf. Records, 1999, section 14.1.

[2] D. A. Paice, "Power Electronic Converter Harmonic Multipulse Methods for Clean Power", New York, IEEE Press, 1996.

[3] S. Choi, P. N. Enjeti, and I. J. Pitel, "Polyphase Transformer Arrangements with Reduced kVA Capacities for Harmonic Current Reduction in Rectifier-Type Utility Interface", in IEEE Trans. on Power Electronics, vol. 11, pp. 680-690, Sep. 1996.

[4] F.J.M. Seixas, and I. Barbi, "A New 12kW ThreePhase 18-Pulse High Power Factor AC-DC Converter with Regulated Output Voltage for Rectifier Units", in INTELEC Conf. Records, 1999, section 14.2.

[5] F.J.M. Seixas, and I. Barbi, "A New 18-Pulse AC-DC Converter with Regulated DC Output and High Power Factor for Three-Phase Applications", In Brazilian Power Electronics Conference Records, 1999, pp. 582587.

[6] C. Niermann, "New Rectifier Circuits with Low Mains Pollution and Additional Low Cost Inverter for Energy Recovery" in: EPE Conf. Records, 1989, pp. 11311136.

[7] C. A. Muñoz, and I. Barbi, "A New High-Power-Factor Three-Phase AC-DC Converter: Analysis, Design, and Experimentation", in IEEE Trans. on Power Electron., vol. 14, no 1, pp. 90-96, Jan. 1999.

[8] F.J.M. Seixas, and I. Barbi, “A New Three-Phase Low 
THD Power Supply with High-Frequency Isolation and 60V/200A Regulated DC Output", in IEEE PESC Conf. Records, Jun. 2001, pp. 1629-1634.

[9] F.J.M. Seixas, and I. Barbi, "A Robust 12kW ThreePhase Rectifier using a 18-Pulse Autotransformer and Isolated DC-DC Converters", in COBEP'01 Records, Nov. 2001, pp. 686-691.

[10] F.J.M. Seixas, " $12 \mathrm{~kW}$ High Power Factor AC-DC Converters Using Autotransformer with Multipulse Differential Connection”, $\mathrm{PhD}$ Thesis (in Portuguese), in UFSC/INEP - Federal University of Santa Catarina, Advisor Prof. Ivo Barbi, Out./2001.

[11] B. Andreycak, "Phase Shifted, Zero Voltage Transition Design Consideration and the UC3875 PWM Controller", Unitrode Corporation, Application Note U136A, 1997.

\section{DADOS BIOGRÁFICOS}

Falcondes José Mendes de Seixas, nascido em Jales-SP em 1965, formou-se em Engenharia Elétrica pela Escola de Engenharia de Lins em 1987. Em 1993 e 2001 recebeu os de fator de potência, conexões especiais de transformadores e qualidade de energia elétrica.

Ivo Barbi, nascido em Gaspar (SC) em 1949, formou-se em Engenharia Elétrica pela Universidade Federal de Santa Catarina - UFSC em 1973. Em 1976 recebeu o título de Mestre pela mesma Universidade e, em 1979, recebeu o título de Doutor pelo Institut National Polytechnique de Toulouse, França. Desde 1974 é professor na UFSC e atualmente é professor titular do Departamento de Engenharia Elétrica. É membro fundador da SOBRAEP tendo sido seu primeiro presidente. Desde 1992, é Editor Associado na área de Conversores de Potência da IEEE Transactions on Industrial Electronics. Suas áreas de atuação compreendem modelagem, análise, projeto e aplicações de conversores estáticos operando em alta freqüência e correção de fator de potência de fontes de alimentação.

títulos de Mestre e de Doutor em engenharia elétrica pela UFSC-Universidade Federal de Santa Catarina, em Florianópolis-SC. Desde 1988 é professor na UNESPUniversidade Estadual Paulista, em Ilha Solteira-SP e atualmente é professor assistente doutor no Departamento de Engenharia Elétrica. Suas áreas de interesse compreendem conversores estáticos operando em alta freqüência, correção 